\title{
Potential Biomarkers for Predicting Bloodstream Bacterial Infection in Tanzanian Children
}

\section{Ng'weina Francis Magitta $^{1,2 *}$ and Meshack Denson Shimwela ${ }^{3}$}

${ }^{1}$ Ifakara Health Institute (IHI), Biomedical and Laboratory Sciences Thematic Group, Dar es Salaam, Tanzania ${ }^{2}$ Department of Biochemistry, Mbeya College of Health and Allied Sciences, University of Dar es Salaam, Mbeya, Tanzania ${ }^{3}$ Department of Internal Medicine, Amana Regional Referral Hospital, Dar es Salaam, Tanzania

*Corresponding Author: Ng'weina Francis Magitta, Ifakara Health Institute (IHI), Biomedical and Laboratory Sciences Thematic Group, Dar es Salaam, Tanzania. E-mail: nmagitta@ihi.or.tz

Received: February 13, 2018; Published: March 20, 2018

DOI: 10.31080/ASMI.2018.01.0037

\begin{abstract}
Background: Bloodstream bacterial infection is a major cause of morbidity and mortality among children in Africa. The existing biomarkers have limited sensitivity and specificity. Irrational use of antibiotics in non-malarial fevers increases healthcare cost, morbidity and accelerate antibiotic resistance.

Objective: To identify potential biomarkers for bloodstream bacterial infection in children for developing a point-of-care diagnostic test in limited-resource setting.

Methods: We obtained blood from children aged less than 5 years with clinically-suspected septicaemia. Blood cultures were performed by BACTEC ${ }^{\mathrm{TM}}$ technique followed by bacterial identification using conventional methods and PCR. Using ELISA we analysed previously reported serum biomarkers for bloodstream bacterial infection; procalcitonin (PCT) and C-reactive protein (CRP), lipopolysaccharide (LPS) and lipoteichoic acid (LTA).

Results: We recruited 79 children, 54\% were male, mean age of 34 months. Almost all patients had fever, with a mean duration of sickness of 3.8 days; $92 \%$ completed age-appropriate vaccines; $67 \%$ received prior antibiotic therapy and $20 \%$ had altered consciousness. Using box plot, LTA displayed the most sensitivity compared to LPS, PCT and CRP. Further, we used receiver-operating characteristic (ROC) curves to evaluate the performance of the markers based on the subset analysis of 25 samples using quantitative PCR results as a reference. The curves displayed similar sensitivity for PCT and LPS with area under the curve (AUC) of 0.69. Consistently, CRP did not perform well in both analyses.
\end{abstract}

Conclusion: Both PCT and LPS showed acceptable diagnostic thresholds for septicaemia. However, large-powered studies are required for validation prior to their use in clinical practice.

Keywords: Biomarkers; Bloodstream Bacterial Infection; Procalcitonin; C-Reactive Protein; Lipopolysaccharide; Lipoteichoic Acid

\section{Background}

There is a remarkable improvement in child survival globally due to multiple interventions. However, overall child mortality remains substantially high particularly in sub-Saharan Africa (SSA) $[1,2]$. Bloodstream bacterial infection is an important cause of morbidity and mortality among children less than 5 years [3]. The emerging resistance to antibiotics in Africa in the context of weak antibiotic resistance stewardship and irrational prescription of antibiotics could contribute to the child mortality [4,5].

The gold standard for the diagnosis of bloodstream bacterial infection involves culture systems coupled with standard identification methods, both of which are not readily available in the majority of health facilities in SSA. A review by Nelson., et al. reports a number of potential biomarkers for bloodstream bacterial infection albeit with limited diagnostic threshold [6]. In routine practice, leukocyte count, erythrocyte sedimentation rate (ESR), and C-reactive protein (CRP) are commonly employed as guides for antibiotic administration. Recently, there has been an increasing number of potential biomarkers for bacterial sepsis, such as pro-adrenomedullin (ProADM), procalcitonin (PCT), lipopolysaccharide (LPS), lipoteichoic acid (LTA), mid-regional pro-atrial natriuretic peptide (ANP), soluble triggering receptor expressed on myeloid cells 1 (sTREM-1), pancreatic stone protein (PSP), a range of pro-inflammatory cytokines (TNF- $\alpha$, IL-6, IL-8, IL-27) and soluble urokinase-type plasminogen activator receptor (suPAR) [6-8].

In the absence of a reliable marker for the diagnosis of bloodstream bacterial infection especially when antibiotics are required to be administered urgently, a collection of clinical and laboratory 
indices are used as guides to the management decision systems. This diagnostic approach has been shown to improve the predictive values of biomarkers with moderate threshold. For instance, a Lab-score consisting of CRP, PCT and urinary dipstick for the identification of severe bacterial infection revealed the sensitivity of $86 \%$ and the specificity $83 \%$ superior to that of any single variable [9]. However, currently, PCR-based techniques offer alternative rapid and highly sensitive diagnostic platforms for bloodstream bacterial infection $[10,11]$. This method is more expensive but offers an opportunity to characterize multiple bacterial isolates at a single setting with high sensitivity and specificity.

Of all the markers currently known, PCT has been shown to be a better predictor and superior to the commonly used clinical variables and laboratory tests in the diagnosis of bloodstream bacterial infection and has been shown to correlate with the extent and severity of microbial infection [12-14]. Currently, many authorities recommends use of PCT as clinical guide for diagnosis of sepsis in emergency situations prior to administration of antibiotic therapy [15]. Moreover, serum levels of PCT correlates well with the degree of infectious load and can safely be used as a guide for dose deescalating or discontinuation of antibiotic use [16]. In order to improve predictive value of markers for bloodstream bacterial infections, one approach is the combination of prediction rules of different tests for clinical and laboratory markers [17]. For instance, LTA and LPS which represents major antigens respectively in Gram positive and Gram negative bacteria, through their pivotal role in the modulation of the systemic inflammatory response syndrome (SIRS) could serve as biomarkers for bacterial infection [18].

In the effort to explore relevant biomarkers, we attempted to characterize four previously identified biomarkers for bloodstream bacterial infection in the local setting. Identification of appropriate biomarkers will provide opportunity for developing a point-of-care rapid diagnostic test for bloodstream bacterial infection for use in limited-resource setting. Additionally, the test will provide a tool for guiding rational use of antibiotics thereby reducing chances for antibiotic resistance.

\section{Patients and Methods}

Patients and study design

In this cross-sectional study, we recruited 79 children aged less than 5 months from three secondary care health facilities in Dar es Salaam, Tanzania. The children were recruited at the point of admission for hospitalization due to clinical suspicion of bloodstream bacterial infection without a primary source. Prior to recruitment all children underwent routine physical examination by experienced registrar and further tailored investigations conducted. All children were tested for malaria by a rapid diagnostic test at the respective health facility and those who tested positive were excluded.

\section{Sampling and recruitment}

We recruited a total of 79 children with clinically-suspected bloodstream bacterial infection admitted at three secondary care health facilities in Dar es Salaam, Tanzania. Of the children admit- ted in the health facility, we recruited children aged between 1 and 60 months with fever and clinical features suggestive of bloodstream bacterial infection. These probable cases of bloodstream bacterial infection underwent testing for malaria using mRDT and those who tested positive were excluded from the study. We classified the remaining mRDT negative cases as likely cases of bloodstream bacterial infection, which we recruited into the study, interviewed for further information and collected blood samples for blood culture and biomarkers analysis. The scheme for sampling is herewith presented in figure 1 below.

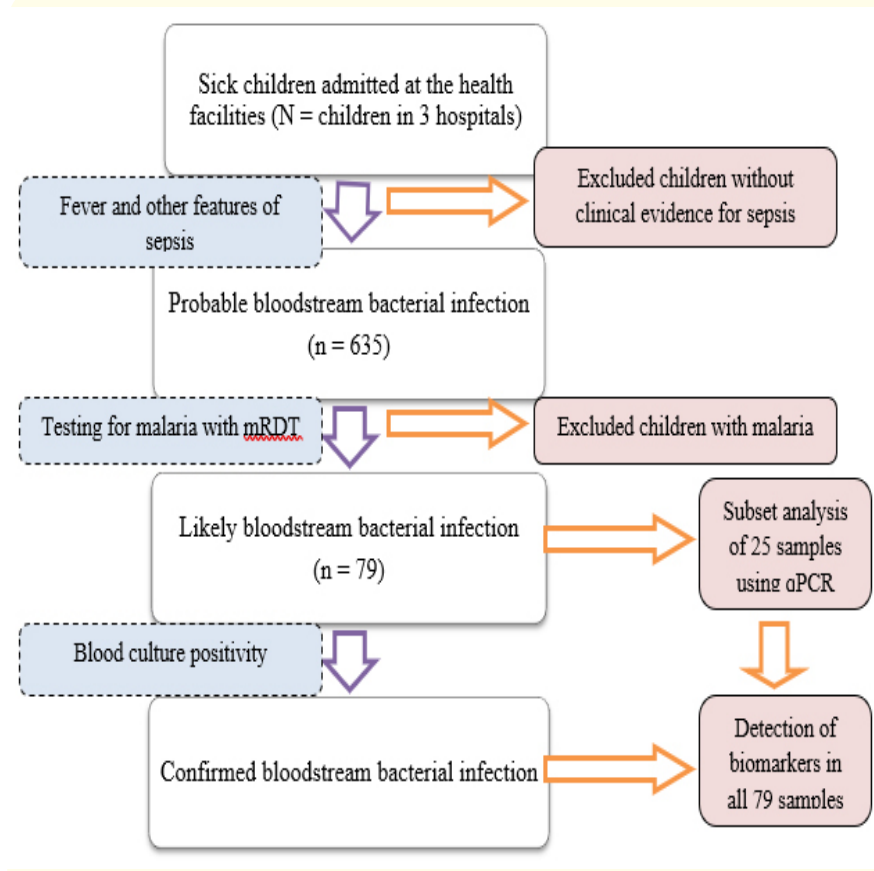

Figure 1: Sampling procedure for children with clinical suspicion of bloodstream bacterial infection.

Data collection

We collected information on basic demographic characteristics and vaccination history for all eligible children. The children, in addition, underwent measurement for anthropometry including height/length, weight and mid-upper arm circumference (MUAC) using standard procedures. Basic demographic information and important clinical characteristics were collected from the informant using a structured questionnaire.

\section{Laboratory methods}

Blood sample collection and handling

About $5 \mathrm{~mL}$ of blood was collected from eligible children for blood culture and identification of isolates using PCR as well as determination of putative biomarkers using human ELISA technique. From the eligible pediatric patient, $2 \mathrm{~mL}$ of blood was collected in BD BACTEC Peds Plus ${ }^{\mathrm{TM}} / \mathrm{F}$ medium (Becton Dickinson, NJ, USA) for culture and mixed thoroughly by inversion. Then, without removing the collection accessories, $3 \mathrm{~mL}$ of blood was drawn into the BD K2EDTA vacutainer tubes (Becton Dickinson, USA) for PCR and biomarker analysis and mixed thoroughly, labelled and immediately placed at $4^{\circ} \mathrm{C}$. Blood samples were transported to the laboratory in specialized containers at $2-8^{\circ} \mathrm{C}$ with temperature monitoring ther- 
mometer. Blood samples from K2EDTA were centrifuged at $800 \mathrm{~g}$ for 15 minutes at $4^{\circ} \mathrm{C}$. At least 6 cryotubes $(0.5 \mathrm{~mL})$ were used for storing plasma samples and 1 eppendorf tube $(1.5 \mathrm{~mL})$ for storing remaining pellet. The aliquot of about $0.3 \mathrm{~mL}$ plasma were separated in each specified tube and put the remaining pellet in eppendorf tube and both tubes were put in the labeled cryoboxes and immediately frozen at $-80^{\circ} \mathrm{C}$.

\section{Blood culture and identification of isolates}

About $2 \mathrm{~mL}$ of blood samples in BD BACTEC vials were directly registered and quickly incubated in the BD BACTEC ${ }^{\mathrm{TM}} 9050$ Blood Culture System (Becton Dickinson, NJ, USA) according to the manufacturer's recommendations. The growths obtained were identified using a combination of conventional bacteriological and API biochemical tests as described elsewhere. Furthermore, in a subset of 25 blood samples, we employed quantitative PCR to identify bacterial isolates as previously described. In this subset analysis, parallel to identification of bacterial pathogens, we employed qPCR for detection of co-infection with plasmodium spp for malaria and fungal infection based on human DNA as internal control as previously described.

\section{Detection of putative biomarkers using ELISA techniques}

Putative biomarkers for bloodstream bacterial infection comprising PCT, CRP, LTA and LPS were determined using competitive inhibition human ELISA techniques. The biomarkers were measured in plasma using commercially available human ELISA kits; CRP and PCT (ABCAM, USA) together with LPS and LTA (Novatein Biosciences, USA) according to the manufacturer's recommendations as previously described $[14,18,19]$. In brief, monoclonal antibodies specific for respective antigen were pre-coated onto a micro-titre plate, which results in a competitive inhibition reaction occurring between biotin-labeled and unlabeled marker. After incubation the unbound conjugate were washed off and the next, avidin conjugated to horse-radish peroxidase (HRP) was added to each micro-titre plate well and incubated. Then, the amount of the bound HRP-conjugate was inversely proportional to the concentration of the marker in the sample. After addition of the substrate solution, the intensity of the color developed is inversely proportional to the concentration of the marker in the sample.

\section{Statistical analysis}

Data were analyzed using STATA statistical package. Demographic variables and basic clinical characteristics together with anthropometry were analyzed as numerical values and percentages. The performance of putative biomarkers as predictors of bloodstream bacterial infection were performed using box plot and receiver operating characteristic (ROC) curves and reported generated as area under the curves (AUC). The later was performed based on a subset of samples with qPCR results as a reference in the model.

\section{Results}

General patients' characteristics and anthropometry

As shown in table 1, a total of 79 children (54\%, males) aged less than 5 years (mean age, 34 months) were recruited into the study. Ninety-nine percent of the children presented with fever and all children had a mean duration of sickness of 3.8 days. Furthermore, it was revealed that $67 \%$ of children were reported to have received prior antibiotic therapy either at the referring health facility or on arrival during hospitalization at the point of recruitment. On physical examination, $20 \%$ of children were found to have altered level of consciousness. Moreover, table 2 indicates that on assessment of the nutritional status of the recruited children, based on anthropometry, a third of the children were found have stunting. Furthermore, based on measurement of MUAC, 20\% of the children were found to have malnutrition. Likewise, about $22 \%$ and $25 \%$ of the children were found to have acute malnutrition and underweight respectively. There was no obvious difference in the nutritional status among different age categories of the study children.

\begin{tabular}{|l|c|}
\hline \multicolumn{1}{|c|}{ Variable } & Statistic (n, \%) \\
\hline Male (months) & $43(54.4)$ \\
\hline Less than 12 months & $27(34.2)$ \\
\hline 12 - 35 months & $25(31.7)$ \\
\hline 36 - 60 months & $27(34.2)$ \\
\hline Presence of fever on admission & $78(98.7)$ \\
\hline Antibiotics given & $53(67.1)$ \\
\hline $\begin{array}{l}\text { Age-appropriate vaccines com- } \\
\text { pleted }\end{array}$ & $73(92.4)$ \\
\hline \multicolumn{1}{|c|}{ Duration of sickness in days } & \\
\hline Mean (SD) & $4.7(3.8)$ \\
\hline \multicolumn{1}{|c|}{ General conditions } & $63(79.8)$ \\
\hline Alert & $12(15.2)$ \\
\hline Semi-conscious & $4(5.1)$ \\
\hline Unconscious &
\end{tabular}

Table 1: Basic demographic and clinical characteristics of children with bloodstream bacterial infection $(\mathrm{N}=79)$.

\begin{tabular}{|l|c|c|c|c|}
\hline Variable (n, \%) & $<\mathbf{1 2} \mathbf{~ m o}$ & $\begin{array}{c}\mathbf{1 2}-\mathbf{3 5} \\
\mathbf{m o}\end{array}$ & $\begin{array}{c}\mathbf{3 6 - 6 0} \\
\mathbf{m o}\end{array}$ & Overall \\
\hline & $\mathrm{n}=27$ & $\mathrm{n}=25$ & $\mathrm{n}=27$ & $\mathrm{~N}=79$ \\
\hline $\begin{array}{l}\text { Stunting } \\
\text { (height/age) }\end{array}$ & $4(14.8)$ & $10(40.0)$ & $\begin{array}{c}11 \\
(40.7)\end{array}$ & $25(31.7)$ \\
\hline $\begin{array}{l}\text { Under-weight } \\
\text { (weight/age) }\end{array}$ & $4(14.8)$ & $8(32.0)$ & $8(29.6)$ & $20(25.3)$ \\
\hline $\begin{array}{l}\text { Acute under- } \\
\text { nutrition } \\
\text { (weight/height) }\end{array}$ & $9(33.3)$ & $6(24.0)$ & $3(11.1)$ & $18(22.8)$ \\
\hline $\begin{array}{l}\text { Malnutrition } \\
\text { (MUAC) }\end{array}$ & $7(25.9)$ & $8(32.0)$ & $1(3.7)$ & $16(20.3)$ \\
\hline
\end{tabular}

Table 2: Anthropometry measurements for all children recruited into the study $(\mathrm{N}=79)$.

Performance of biomarkers in the prediction of bloodstream bacterial infection

Based on box plot as depicted in figure 2, the median for LTA was higher than those of PCT and LPS. The graphs for the three markers overlapped especially between lower and upper quartiles. The upper quartile for PCT was the same as the lower quartile for LTA, and higher than that of LPS. It was highlighted that 
the inter-quartile range for LPS was the smallest of all the three markers. Overall, it was observed that LTA displayed the most sensitivity compared to LPS, PCT and CRP. Furthermore, as highlighted in figure 3, using ROC curves to evaluate the performance of the markers based on the subset analysis of 25 samples using PCR results as a reference. From ROC curve analysis, it was evident that both PCT and LPS displayed similar sensitivity for predicting bloodstream bacterial infection in children, with AUC of about $69 \%$ for both markers.

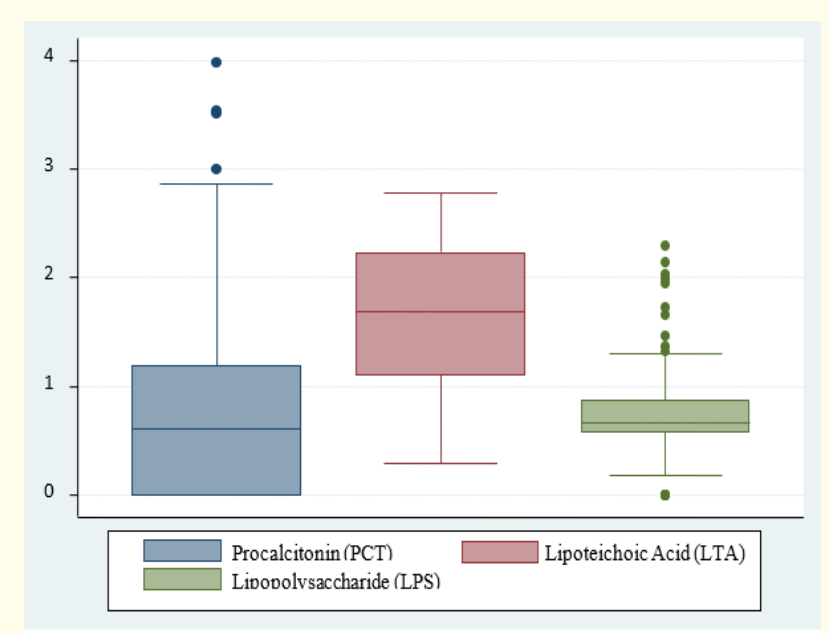

Figure 2: The comparison of the predictive values of three sensitive biomarkers for bloodstream bacterial infection in children based on all 79 samples

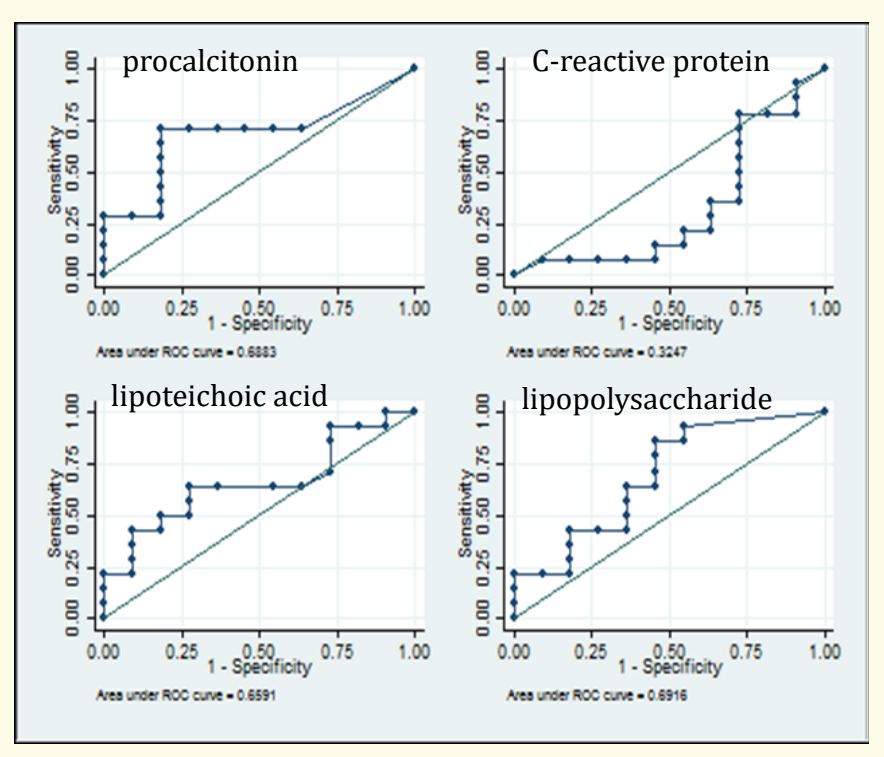

Figure 3: ROC curves for four investigated biomarkers of bloodstream bacterial infection in children based on the subset analysis of 25 samples with PCR results as a reference.

\section{Discussion}

The investigators analyzed four predetermined biomarkers for bloodstream bacterial infection in children in Tanzania and evaluated their predictive value for use as a rapid diagnostic test. The preliminary findings indicate improved diagnostic value of LTA as compared to other markers. However, using a subset analysis with quantitative PCR as the reference it was observed that PCT and LPS were more sensitive markers for prediction of bloodstream bac- terial infection while CRP consistently performed poorly in both analyses.

Our results are in line with other previous studies indicating that PCT could be a promising marker for bloodstream bacterial infection $[7,19]$. Though it did not show any remarkable predictive value in our study, CRP is a well-documented marker of bacterial sepsis and has been in clinical use for a considerable period of time. Generally, serum levels of biomarkers tend to decrease beyond detectable ranges in patients who have received prior antibiotic therapy [20]. This could have potentially affected the performance of the four analyzed biomarkers resulting into inconclusive evidence. However, lack of adequate predictive value for PCT and other markers may be improved by designing a collection of biomarkers with moderate thresholds into a single diagnostic "multistix"; the approach that has been similarly applied in other conditions including the diagnosis of spontaneous bacterial peritonitis [21].

PCT together with other related proteins are constitutively expressed from CALC-1 gene in all cell types during systemic inflammation resulting from bacterial infection [15]. The detailed characterization of the kinetic profile of CALC-1 gene products, other than PCT, may provide better insight on the potential role of PCT and related proteins in the prediction of bloodstream bacterial infection. The role of LTA and LPS as markers for bloodstream bacterial infection should be interpreted with caution since their usefulness depends on the nature of the cell walls of the responsible bacterial pathogens. In this regard, the two markers should always complement general biomarkers, serving as markers specific to either Gram positive or negative and provide, yet, an improved guide to the selection of a class of antibiotic therapy. However, though not of bacterial source, previous studies have reported that significantly high level of PCT can differentiate Gram negative from Gram positive bacterial infection $[12,22]$.

It is important to highlight the possible limitations of this study. For instance, it was anticipated to recruit children with suspected bloodstream bacterial infection preferably without prior treatment with antibiotics. However, it was observed that over twothird of the children recruited at secondary health facilities had already received antibiotics. This practice could influence negative blood culture results which were initially considered as gold standard and thus reference point for the analysis of the performance of the biomarkers. In addition, it may not be possible to anticipate the influence the antibiotics might have on the expression and half-life of specific biomarkers in blood [16,20].

Due to this clinical pitfall, and lack of adequate funds, the evaluation of performance of biomarkers was therefore analyzed based on qPCR results for only a third of samples. This sample size was inadequate to reach a plausible inference and conclusion, thus, it is likely that small sample size could have a negative impact on the power of the study.

\section{Conclusion}

Bloodstream bacterial infection is a major problem among under-fives in Tanzania. However, the diagnosis of bloodstream bacterial infection is a major challenge due to lack of a reliable rapid test. The study revealed that PCT could be a potential biomarker for rapid diagnosis of bloodstream bacterial infection in children. It is further suggested that the combination of PCT with other 
biomarkers with moderate threshold could improve its diagnostic performance. We recommend that large powered studies of better designs encompassing control groups preferably recruiting children who have not received prior antibiotic therapy are required to validate these markers. Furthermore, attempts should be made to conduct a detailed studies on the characterization of PCT and other splice variants derived from CALC-1 gene for better insight on their diagnostic potential.

\section{Authors Contributions}

NFM conceived and designed the study and MDS reviewed the draft of the study. NFM prepared the results and wrote the first draft of the manuscript. MDS reviewed the final version of the manuscript.

\section{Acknowledgement}

This study was conducted by Ifakara Health Institute in collaboration with local partners. This work was supported through funds obtained from Grand Challenges Canada - Stars in Global Health. We thank Mr. Tedson Lukindo for performing the laboratory analysis and Dr. Abdallah Mkopi for his assistance in data analysis. We thank all research assistants together with administrative and health authorities in all three hospitals - Amana, Mwananyamala and Temeke in Dar es Salaam, Tanzania. We are greatly indebted to all children and their parents involved in the study.

\section{Bibliography}

1. Wardlaw T., et al. "UNICEF Report: enormous progress in child survival but greater focus on newborns urgently needed". Reproductive Health 11 (2014): 82.

2. Wardlaw T., et al. "Child survival: a message of hope but a call for renewed commitment in UNICEF report". Reproductive Health 10 (2013): 64.

3. Liu L., et al. "Global, regional, and national causes of child mortality: an updated systematic analysis for 2010 with time trends since 2000". Lancet 379.9832 (2012): 2151-2161.

4. Essack SY., et al. "Antimicrobial resistance in the WHO African region: current status and roadmap for action". Journal of Public Health 39.1 (2017): 8-13.

5. Tadesse BT., et al. "Antimicrobial resistance in Africa: a systematic review". BMC Infectious Diseases 17.1 (2016): 616.

6. Nelson GE., et al. "Biomarkers for sepsis: a review with special attention to India". BioMed Research International (2014).

7. Harikrishna AM., et al. "Biomarkers for the diagnosis of bacterial infections: in pursuit of the 'Holy Grail'. Indian Journal of Medical Research 141.3 (2015): 271-273.

8. Hanna WJ., et al. "Interleukin-27: a novel biomarker in predicting bacterial infection among the critically ill”. Critical Care 19 (2015): 378.
9. Galetto-Lacour A., et al. "Validation of a laboratory risk index score for the identification of severe bacterial infection in children with fever without source". Archives of Disease in Childhood 95.12 (2010): 968-973.

10. Lucignano B., et al. "Multiplex PCR allows rapid and accurate diagnosis of bloodstream infections in newborns and children with suspected sepsis". Journal of Clinical Microbiology 49.6 (2011): 2252-2258.

11. Opota O., et al. "Microbial diagnosis of bloodstream infection: towards molecular diagnosis directly from blood". Clinical Microbiology and Infection 21.4 (2015): 323-331.

12. Friend KE., et al. "Procalcitonin elevation suggests a septic source". American Surgeon 80.9 (2014): 906-909.

13. Hoeboer S H., et al. "Old and new biomarkers for predicting high and low risk microbial infection in critically ill patients with new onset fever: a case for procalcitonin". Journal of Infection 64.5 (2012): 484-493.

14. Mahajan P., et al. "Procalcitonin as a marker of serious bacterial infections in febrile children younger than 3 years old". Academic Emergency Medicine 21.2 (2014): 171-179.

15. Khan MJ and Adil I Khan. "Procalcitonin: Uses in the Clinical Laboratory for the Diagnosis of Sepsis". Laboratory Medicine 41.3 (2015): 173-177.

16. Rhee C. "Using Procalcitonin to Guide Antibiotic Therapy". Open Forum Infectious Diseases 4.1 (2017).

17. Irwin AD., et al. "Novel biomarker combination improves the diagnosis of serious bacterial infections in Malawian children". BMC Medical Genomics 5 (2012): 13.

18. Triantafilou M., et al. "Serum proteins modulate lipopolysaccharide and lipoteichoic acid-induced activation and contribute to the clinical outcome of sepsis". Virulence 3.2 (2012): 136-145.

19. Vijayan AL., et al. Procalcitonin: a promising diagnostic marker for sepsis and antibiotic therapy. Journal of Intensive Care 5 (2017): 51.

20. Viallon A., et al. "Decrease in serum procalcitonin levels over time during treatment of acute bacterial meningitis". Critical Care 9.4 (2005): R344-350.

21. Nousbaum JB., et al. "Diagnostic accuracy of the Multistix 8 SG reagent strip in diagnosis of spontaneous bacterial peritonitis". Hepatology 45.5 (2007): 1275-1281.

22. Guo SY., et al. "Procalcitonin is a marker of gram-negative bacteremia in patients with sepsis". American Journal of the Medical Sciences 349.6 (2015): 499-504.

\section{Volume 1 Issue 4 April 2018}

(C) All rights are reserved by Ng'weina Francis Magitta and Meshack Denson Shimwela. 